\title{
Critical test of the interaction of surface plasmon resonances with molecular vibrational transitions
}

\author{
Edward Sachet ${ }^{1}$, D. E. Aspnes ${ }^{2}$, J.-P. Maria ${ }^{3}$, Stefan Franzen*4
}

1. Third Floor Materials, Inc., Raleigh, NC 27603

2. Department of Physics, North Carolina State University, Raleigh, NC 27695

3. Department of Materials Science, North Carolina State University, Raleigh, NC 27695

4. Department of Chemistry, North Carolina State University, Raleigh, NC 27695, franzen@ncsu.edu

\section{Supporting Information}

\section{S1. Three-layer Fresnel model for reflection and transmission}

Calculations based on the Airy Equation give excellent agreement with observations of the reflected infrared radiation both in an empty cell and in the presence of $\mathrm{N}_{2} \mathrm{O}$ gas. The three-layer model gives complex reflectances $r_{\text {soa }}$ in the three-phase (substrate/overlayer/ambient) geometry. This equation is

$$
r_{\text {soa }}=\frac{r_{o a}+r_{s o} e^{2 i k_{o \perp} d}}{1+r_{o a} r_{s o} e^{2 i k_{o \perp} d}}
$$

where the subscripts a, o, and s represent the ambient $\left(\mathrm{CaF}_{2}\right.$ prism/glass), overlayer ( $\mathrm{CdO}$ film), and substrate $\left(\mathrm{N}_{2} \mathrm{O}\right.$ gas $)$, respectively. For TE polarization

$$
r_{o a}^{T E}=\frac{n_{a \perp}-n_{o \perp}}{n_{a \perp}+n_{o \perp}} ; r_{s o}^{T E}=\frac{n_{o \perp}-n_{s \perp}}{n_{o \perp}+n_{s \perp}}
$$

and for TM polarization

$$
r_{o a}^{T M}=\frac{\varepsilon_{o} n_{a \perp}-\varepsilon_{a} n_{o \perp}}{\varepsilon_{o} n_{a \perp}+\varepsilon_{a} n_{o \perp}} ; r_{s o}^{T M}=\frac{\varepsilon_{s} n_{o \perp}-\varepsilon_{o} n_{s \perp}}{\varepsilon_{s} n_{o \perp}+\varepsilon_{o} n_{s \perp}} ;
$$

where

$$
n_{a \perp}=\sqrt{\varepsilon_{a}-\varepsilon_{a} \sin ^{2} \theta} ; n_{o \perp}=\sqrt{\varepsilon_{o}-\varepsilon_{a} \sin ^{2} \theta} ; n_{s \perp}=\sqrt{\varepsilon_{s}-\varepsilon_{a} \sin ^{2} \theta} ; \quad(S 1 f-h)
$$

where $\theta$ is the angle of incidence on the ambient side of the $\mathrm{CdO}$ film.

\section{S2. Derivation of the dispersion relation from the assumption of a plasma resonance}

The reflection coefficient for the two-layer problem is: 


$$
r_{s o}=\frac{\varepsilon_{s} n_{o \perp}-\varepsilon_{o} n_{s \perp}}{\varepsilon_{s} n_{o \perp}+\varepsilon_{o} n_{s \perp}} .
$$

The plasmon will be observed for the condition that the denominator is equal to zero:

$$
\varepsilon_{s} n_{o \perp}+\varepsilon_{a} n_{o \perp}=0
$$

Noting that $n_{j \perp}=\sqrt{\varepsilon_{j}-\varepsilon_{o} \sin ^{2} \theta_{o}}$ we have

$\varepsilon_{o} \varepsilon_{s}\left(\varepsilon_{s}-\varepsilon_{o}\right)=\varepsilon_{o} \varepsilon_{s}^{2}\left(\varepsilon_{s}^{2}-\varepsilon_{o}^{2}\right) \sin ^{2} \theta_{o}$

Thus

$\sin ^{2} \theta_{o}=\frac{\varepsilon_{s}}{\left(\varepsilon_{s}+\varepsilon_{o}\right)}$

which implies that

$\cos ^{2} \theta_{o}=\frac{\varepsilon_{a}}{\left(\varepsilon_{s}+\varepsilon_{o}\right)}$

Using the definition

$\sin ^{2} \theta_{o}=\frac{k_{o x}^{2}}{k_{o}^{2}}$

the dispersion relation requires

$\frac{c^{2}}{\omega^{2}}\left(k_{o x}^{2}+k_{o z}^{2}\right)=\frac{c^{2}}{\omega^{2}} k_{o}^{2}=\varepsilon_{o}$.

Noting that $k_{o x}=k_{s x}=k_{x}$ we can deduce the dispersion relation from the condition that the denominator should vanish:

$$
k_{x}=\frac{\omega}{c} \sqrt{\frac{\varepsilon_{o} \varepsilon_{s}}{\left(\varepsilon_{s}+\varepsilon_{o}\right)}}
$$

The dispersion relation is well known in SPR studies on Au and Ag thin films. In this study, the overlayer dielectric function is given by a free electron model as is appropriate for a metal oxide degenerate semi-conductor such as CdO:Dy, in which a low doping concentration of Dy can support a sufficient charge carrier density to support an infrared SPP. 


\section{S3. Derivation of a dielectric function for a polar gas substrate}

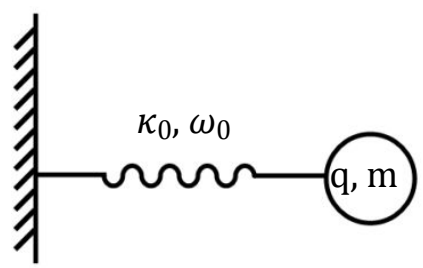

\section{Figure S1. Anisotropic bond model}

The dielectric function for the plasma in a conductor is well known and we have given the results for the Drude model in the text. In order to treat the $\mathrm{N}_{2} \mathrm{O}$ substrate we begin with the equations of motion of anisotropic bond polarizability model applied to a charge moving in response to an applied field. The model for molecular oscillations shown in Figure $\mathbf{S 1}$ is based on a particle (usually an electron) with a mass $\mathrm{m}$ and a charge $\mathrm{q}$, bonded to an immovable system (the nuclei in the molecule or metal) by a spring (chemical bond in the molecule), which has a harmonic force constant $\kappa$. The charge represents the change in dipole moment that arises as a molecule vibrates, a necessary condition for absorption of infrared light. The particle is oscillating at a frequency $\omega_{0}$ which represents the vibrational frequency of a molecule.

$$
\vec{r}_{\text {free }}(t)=\vec{r}_{0}+\Delta \vec{r}_{0} e^{-i \omega_{0} t}
$$

where $\vec{r}_{0}$ is the equilibrium position.

The equation of motion has the following terms: The harmonic restoring force, and the damping friction, which is proportional to the velocity of the motion. Note that the damping friction and the restoring force always has a direction opposite that of the velocity:

$$
\begin{gathered}
\vec{F}=m \frac{d^{2} \vec{r}_{f r e e}(t)}{d t^{2}}=-m \gamma \frac{d \vec{r}_{f r e e}(t)}{d t}-\kappa_{0}\left(\vec{r}_{f r e e}-\vec{r}_{0}\right) \\
-m \omega_{0}^{2} \Delta \vec{r}_{0} e^{-i \omega_{0} t}=i m \omega_{0} \gamma \Delta \vec{r}_{0} e^{-i \omega_{0} t}-\kappa_{0} \Delta \vec{r}_{0} e^{-i \omega_{0} t}
\end{gathered}
$$

Solving Eqn. (2) we obtain:

$$
\kappa_{0}=m \omega_{0}^{2}+i m \omega_{0} \gamma
$$


The particle is driven by the incident electric field, $\vec{E}=\vec{E}_{0} e^{-i \omega t}$ (Figure S2). Hence, the position vector is described by:

$$
\vec{r}(t)=\vec{r}_{0}+\Delta \vec{r} e^{-i \omega t}
$$

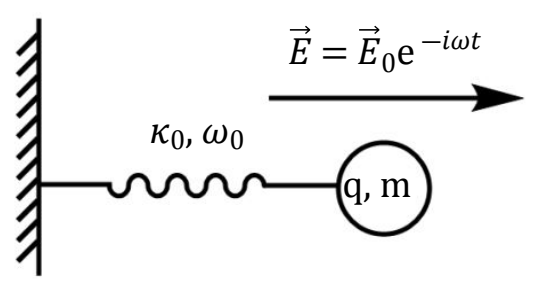

Figure S2. Anisotropic bond model, with time-harmonic driving force.

The equation of motion has a remaining Coulomb force term:

$$
\begin{gathered}
\vec{F}=m \frac{d^{2} \vec{r}(t)}{d t^{2}}=q \vec{E}_{o} e^{-i \omega t}-\kappa_{0}\left(\vec{r}-\vec{r}_{0}\right) \\
-m \omega^{2} \Delta \vec{r} e^{-i \omega t}=q \vec{E}_{o} e^{-i \omega t}-\kappa_{0} \Delta \vec{r} e^{-i \omega t}
\end{gathered}
$$

Solving Eqn. (2) we obtain:

$$
\Delta \vec{r}=\frac{q \vec{E}_{o}}{\kappa_{0}-m \omega^{2}}
$$

Recognizing that the bond force constant is $\kappa_{0}=m \omega_{0}^{2}+i m \omega_{0} \gamma$, the dipole created by the incident field is:

$$
\vec{p}=q \Delta \vec{r}=\frac{q^{2}}{m\left(\omega_{0}^{2}-\omega^{2}\right)+i m \omega_{0} \gamma} \vec{E}_{0}
$$

From this equation we find the Lorentz definition of polarizability:

$$
\alpha=\frac{\vec{p}}{\vec{E}_{0}}=\frac{q \Delta \vec{r}}{\vec{E}_{0}}=\frac{q^{2}}{m\left(\omega_{0}^{2}-\omega^{2}\right)+i m \omega_{0} \gamma}
$$

The polarizability is related to the dielectric susceptibility as

$$
\chi(\omega)=\frac{n \alpha(\omega)}{\varepsilon_{0}}=\frac{n q^{2}}{\varepsilon_{0} m} \frac{1}{\left(\omega_{0}^{2}-\omega^{2}\right)+i \omega_{0} \gamma}
$$


The dielectric susceptibility is in turn related to the dielectric function

$$
\begin{gathered}
\chi(\omega)=1-\varepsilon_{r}(\omega) \\
\varepsilon_{r}(\omega)=1-\chi(\omega)
\end{gathered}
$$

In metals one often interprets the prefactor in terms of a plasma frequency.

$$
\omega_{p}^{2}=\frac{n q^{2}}{\varepsilon_{0} m}
$$

The effect of the field in a metal is to cause as oscillation of the conducting electrons. However, in a gas of polar molecules undergoing molecular vibrations the result of the field is to induce transitions between different states with rate constant $\gamma$. The transitions can be upward (absorption) or downward (stimulated emission). Thus, for the polar gas we use

$$
\gamma^{2}=\frac{n q^{2}}{\varepsilon_{0} m}
$$

Using the definition in S15 the susceptibility in S12 becomes

$$
\chi(\omega)=\frac{\gamma^{2}}{\left(\omega_{0}^{2}-\omega^{2}\right)+i \omega_{0} \gamma},
$$

which has real and imaginary contributions

$$
\chi(\omega)=\frac{\gamma^{2}\left(\omega_{0}^{2}-\omega^{2}\right)}{\left(\omega_{0}^{2}-\omega^{2}\right)^{2}+\omega_{0}^{2} \gamma^{2}}-i \frac{\omega_{0} \gamma^{3}}{\left(\omega_{0}^{2}-\omega^{2}\right)^{2}+\omega_{0}^{2} \gamma^{2}}
$$

such that the dispersion and absorption contributions are both present.

$$
\varepsilon_{r}(\omega)=\varepsilon_{r}^{\prime}(\omega)+\varepsilon_{r}^{\prime \prime}(\omega)
$$

The real part of the dielectric function gives the dispersive response of the gas: 


$$
\varepsilon_{r}^{\prime}(\omega)=1-N \frac{\gamma^{2}\left(\omega_{0}^{2}-\omega^{2}\right)}{\left(\omega_{0}^{2}-\omega^{2}\right)^{2}+\omega_{0}^{2} \gamma^{2}}
$$

where we have added a number density $N$. The imaginary part of the dielectric function gives the absorptive response of the gas:

$$
\varepsilon_{r}^{\prime \prime}(\omega)=N \frac{\omega_{0} \gamma^{3}}{\left(\omega_{0}^{2}-\omega^{2}\right)^{2}+\omega_{0}^{2} \gamma^{2}}
$$

We can compare this model with the standard Lorentizan derived from a response function applied to the excited stated relaxation of the polar gas molecule vibrational state. In that case the Lorentzian function, which replaces a delta function in the first order time-dependent perturbation theory approach to the rate of relaxation, is

$$
\varepsilon_{\mathcal{L}}(\omega)=1-N_{\mathcal{L}} \frac{\gamma_{\mathcal{L}}\left(\omega_{0}-\omega\right)}{\left(\omega_{0}-\omega\right)^{2}+\gamma_{\mathcal{L}}^{2}}+i N_{\mathcal{L}} \frac{\gamma_{\mathcal{L}}^{2}}{\left(\omega_{0}-\omega\right)^{2}+\gamma_{\mathcal{L}}^{2}}
$$

The comparison is shown in the following figures. The value of $\omega_{0}=2500$ is used in both, but the values of $\gamma$ differ in the two cases: $\gamma_{\mathcal{L}}=20 \mathrm{~cm}^{-1}$ and $\gamma=10 \mathrm{~cm}^{-1}$. The values of $N$ used in the figures are $N=70$ in the new model and $N_{\mathcal{L}}=0.56$ in the Lorentzian. These values were chosen for comparison and to permit both absorptive and dispersive parts of the function to be presented on the same plot. It is clear that the two models are superimposable with these adjustments.
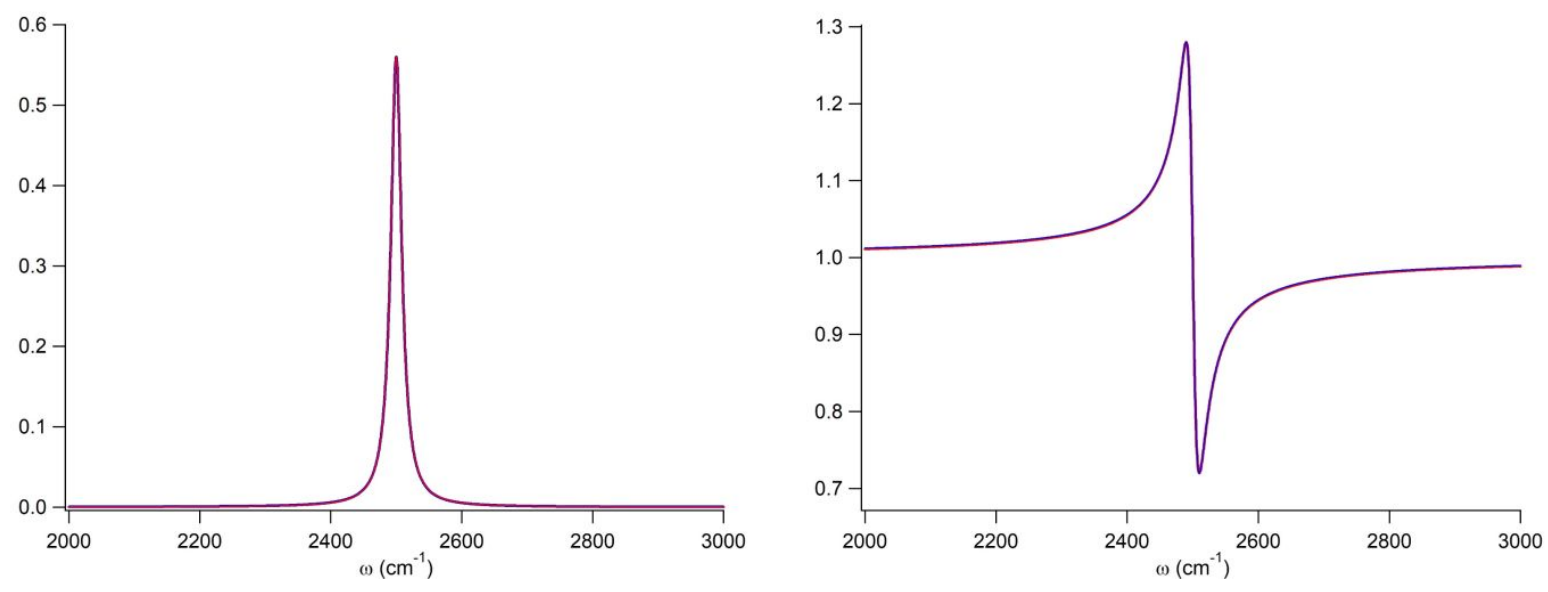
Figure S3. Overlay of dielectric functions calculated using the anisotropic bond model and the standard Lorentzian lineshape, showing that the models superimpose with $\gamma_{\mathcal{L}}=2 \boldsymbol{\gamma}$ and $N=125 N_{\mathcal{L}}$.

\section{S4. A top-down view of the angle-wavenumber map presentation of the data}

The experimental SPR maps in the manuscript were obtained on three different thin films. These are (a) a conducting film of thickness greater than the skin depth, $190 \mathrm{~nm}$ thick, $\mathrm{n}=3.5 \mathrm{x}$ $10^{20} \mathrm{~cm}^{-3}$. This film can support a SPP on resonance with $\mathrm{N}_{2} \mathrm{O}$ vibration. At $10 \mathrm{~nm}$ film (b) is much thinner than the skin depth and cannot support a SPP. Film (c) is thicker than the skin depth but with a lower carrier density so its plasmon is shifted below resonance with the $\mathrm{N}_{2} \mathrm{O}$ vibration, $450 \mathrm{~nm}$. Here $\mathrm{n}=7.0 \times 10^{19} \mathrm{~cm}^{-3}$.

\section{A. The SPP limit on resonance}

In the limit shown in Fig. $3 \mathrm{~A}$, the interface plasmon occurs when the denominator of $r_{s o}$ vanishes. In this case, the SPR given by the Airy equation can be approximated by the dispersion relation at the o-s boundary. In this limit $r_{s o} \gg r_{o a}$, and the problem reduces to a two-layer problem with a reflection coefficient

$$
r_{s o}=\frac{\varepsilon_{s} n_{o \perp}-\varepsilon_{o} n_{s \perp}}{\varepsilon_{S} n_{o \perp}+\varepsilon_{o} n_{s \perp}}
$$

In the Supporting Information we demonstrate that the dispersion relation

$$
k_{x}=\frac{\omega}{c} \sqrt{\frac{\varepsilon_{o} \varepsilon_{s}}{\left(\varepsilon_{s}+\varepsilon_{o}\right)}}
$$

can be derived from the condition that the denominator of Eq. S4 vanishes when a plasma resonance is observed. This wave vector results from an in-phase interaction of the incident electric field with the conduction electrons. Although the dielectric functions $\varepsilon_{s}$ and $\varepsilon_{o}$ are complex, the real or in-phase dispersive part dominates for the overlayer along the plasmon dispersion curve given by Eq. (S5).

\section{B. The ATR limit due to a thin conducting overlayer}

If the overlayer (conducting) layer is very thin, as shown in Figs. 3B and 4B, we can approximate the interface as a two-layer system involving only the ambient and substrate. Since $\varepsilon_{S}$ 
$<\varepsilon_{a}$, this is an example of ATR spectroscopy. Thus, using the definition of the reflection coefficient

$$
r_{s a}=\frac{\varepsilon_{s} n_{a \perp}-\varepsilon_{a} n_{s \perp}}{\varepsilon_{s} n_{a \perp}+\varepsilon_{a} n_{s \perp}}
$$

the observed optical response depends on the absorption by $\mathrm{N}_{2} \mathrm{O}$ within the evanescent field. In this limit the $z$ component of the wave vector is

$$
k_{S Z}=\frac{\omega}{c} \sqrt{\frac{-\varepsilon_{s}^{2}}{\left(\varepsilon_{s}+\varepsilon_{a}\right)}} .
$$

In this case the only coupling of the molecular transition with the radiation field involves the outof-phase (absorptive) response due to the evanescent wave, except when $\varepsilon_{o}$ is replaced by $\varepsilon_{a}$. The in-phase $k_{x}$ response is negligible because the film is optically too thin to behave as a bulk conductor. A similar conclusion is reached for regions of Figs. 3A or $4 \mathrm{~A}$ that are not in resonance with the plasmon dispersion curve.

\section{The ATR limit due to off resonance SPP}

In the limit shown in Fig. 2C, the Airy equation can be approximated in the same way as in Eqs. (S6) and (S7). However, since $\omega_{s p}<\omega_{\mathrm{N}_{2} \mathrm{O}}$ there is no interaction between the SPP and the molecular transition moment of $\mathrm{N}_{2} \mathrm{O}$. There is still a contribution from the ATR precisely as in Eq. (S7). However, this contribution should be exceedingly small, since the intensity of the field reaching the gas molecules is significantly attenuated because of the greater thickness of the overlayer. In this case, the in-phase $k_{x}$ response is negligible because the film behaves as an insulator at the wavenumber $\omega_{\mathrm{N}_{2} \mathrm{O}}$ of $\mathrm{N}_{2} \mathrm{O}$ absorption.

The top-down view of the thin films using an angle-wavenumber map reveals that only film A has a plasmon dispersion curve and that this is the only film that can give rise to coupling of the infrared SPP with molecular transitions. We next consider the enhancement effects possible from the interaction between the SPP and molecular rovibrational transitions using expansions of the Airy equation and different views of the vertical cross-sections of the molecular spectra.

\section{S5. Discussion of the rovibrational spectrum of $\mathrm{N}_{2} \mathrm{O}$}


The model used to simulate the interaction of $\mathrm{N}_{2} \mathrm{O}$ and a mid-IR plasma resonance is designed for relatively low resolution to match that of the experiment. Because of the limited resolution of the IR-VASE, observation of the individual bands shown below is not possible. Rather, one sees the overall envelop of their shape. The $\mathrm{N}_{2} \mathrm{O}$ band was modeled using a method for representing asymmetric line shapes ${ }^{1}$ based on data available in the HITRAN database. ${ }^{2}$

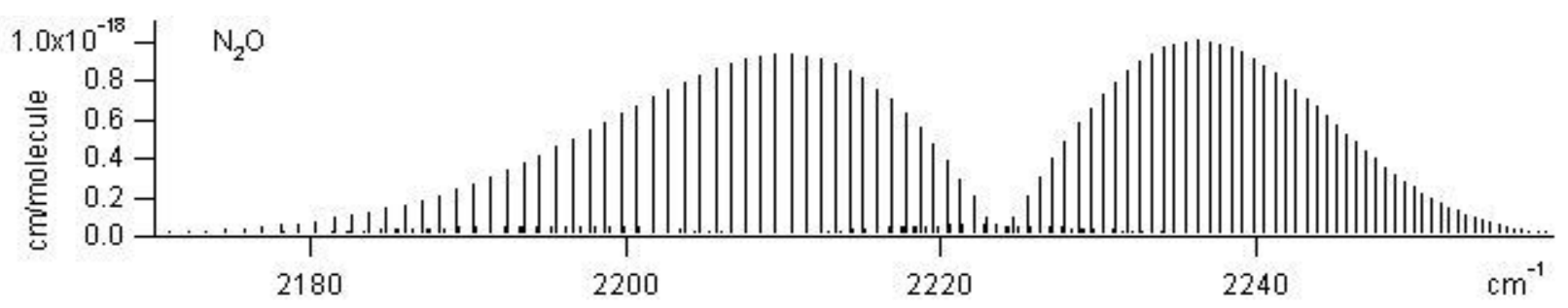

Figure S4. Absorption cross section of $\mathrm{N}_{2} \mathrm{O}$ from the HITRAN 2004 database. For this study we model the envelope of the branches, because the resolution of our experiments is limited to $1 \mathrm{~cm}^{-1}$

\section{S6. Full range reflectance maps of samples $A$ and $C$}
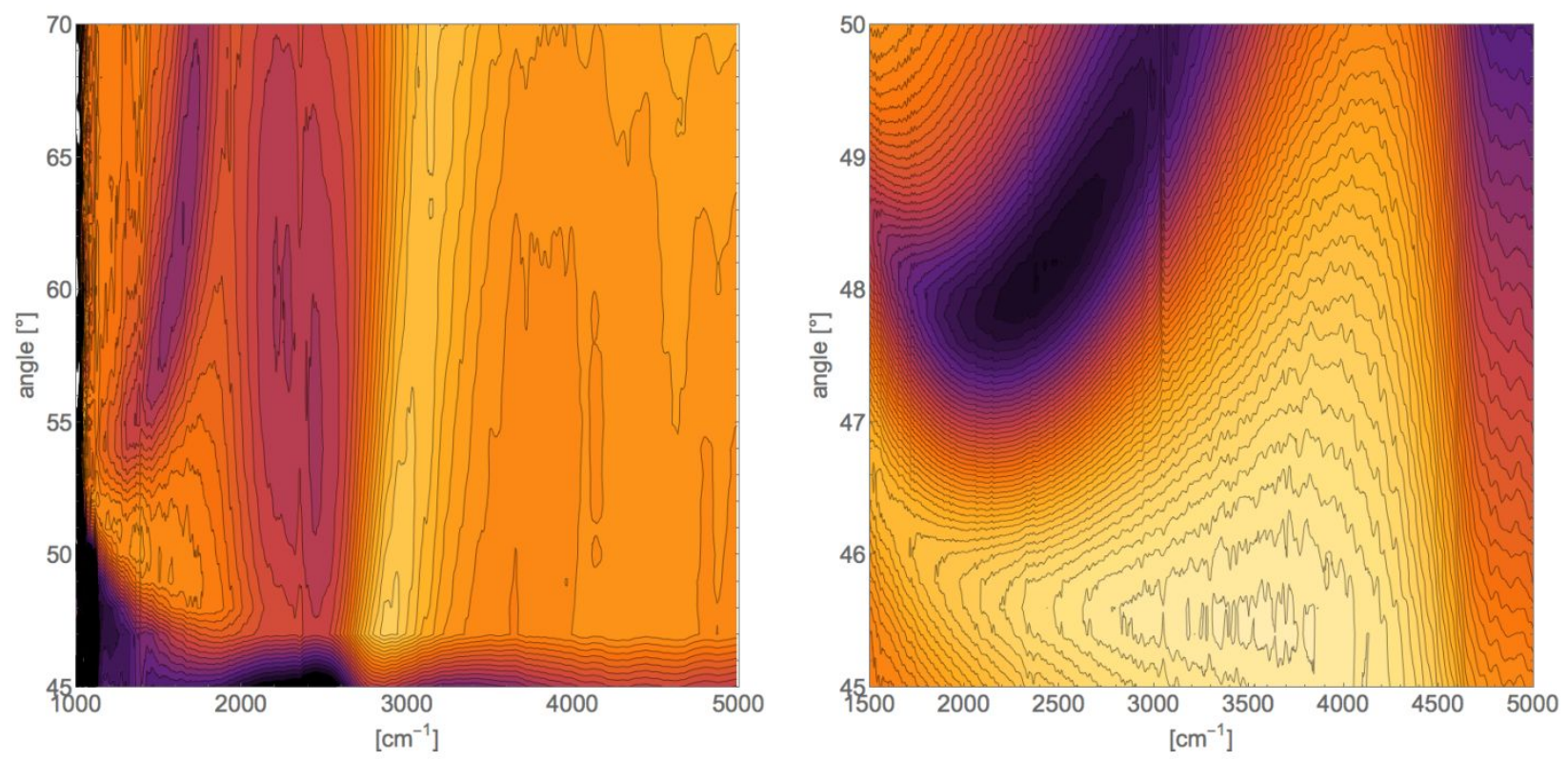

Figure S5. SPP dispersions for samples A (right) and C (left) are depicted in this figure from $1000-5000 \mathrm{~cm}^{-1}$ and incident angles from $45^{\circ}-70^{\circ}$. For $A$, the full SPP dispersion can be seen ranging from $1500-3300 \mathrm{~cm}^{-1}$, with an asymptotic value of $\sim 3100 \mathrm{~cm}^{-1}$ so that $\omega_{s p}>\omega_{N_{2}} O$. From this wider range map, it becomes evident that sample $C$ supports a SPP confined below $2000 \mathrm{~cm}^{-1}\left(50-70^{\circ}\right)$. Its asymptotic value of $\sim 1800 \mathrm{~cm}^{-1} \mathrm{does} \mathrm{not}^{-18}$ intersect with the $\mathrm{N}_{2} \mathrm{O}$ absorption band. Thus, sample $\mathrm{C}$ is an accurate example of the case where $\omega_{s p}<$ $\omega_{N_{2}} \mathrm{O}$ 
S7. $\mathrm{N}_{2} \mathrm{O} / \mathrm{SPP}$ interaction, pressure series 

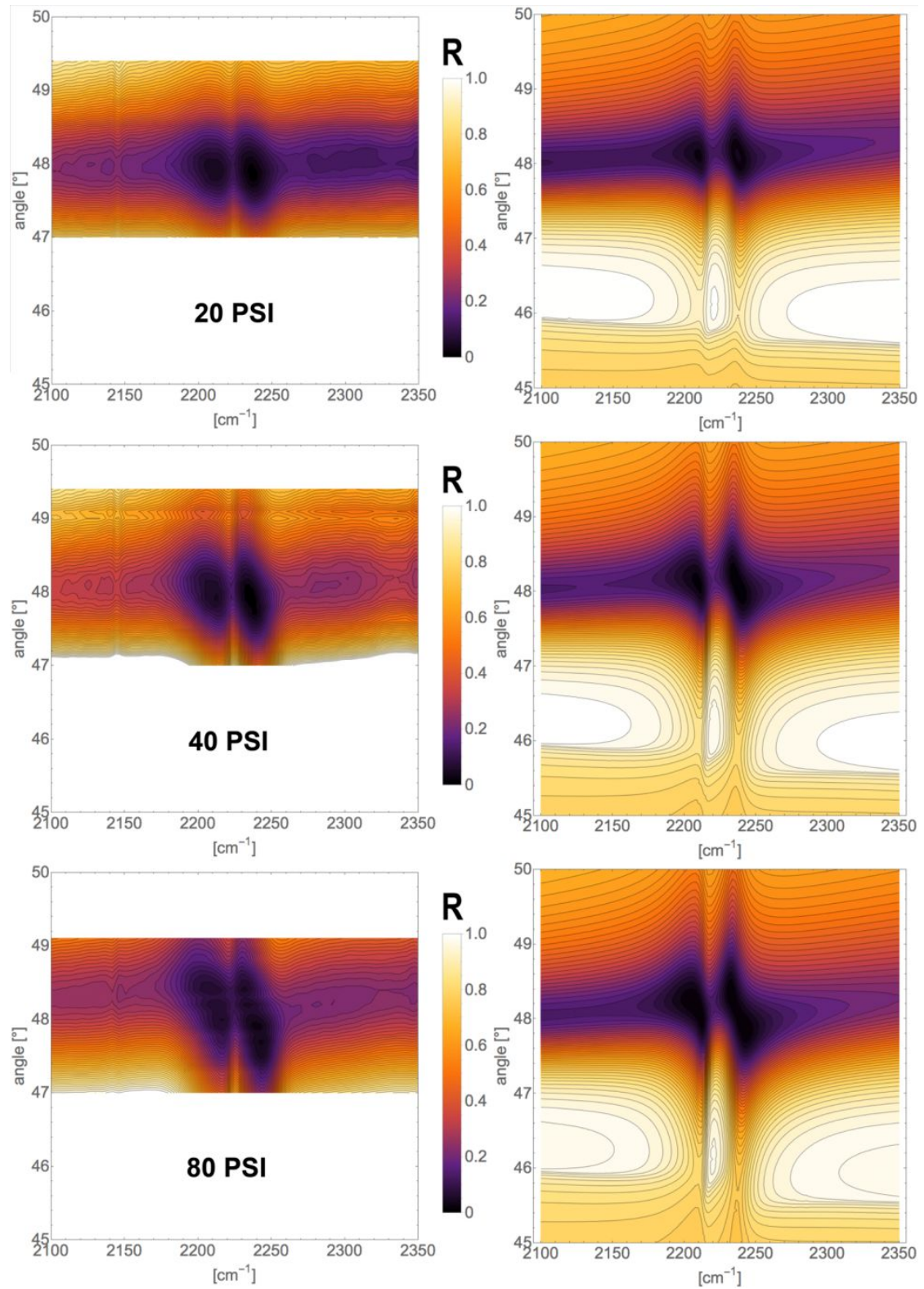

Figure S6. Pressure series. Experimental (left) and simulated (right) reflectance maps of the CdO:Dy SPP coupling to the $\mathrm{N}_{2} \mathrm{O}$ absorption band. With increasing $\mathrm{N}_{2} \mathrm{O}$ pressure, the dispersive line shape of the SPP becomes more apparent, indicating stronger coupling of the SPP to the molecular vibration as the concentration (pressure) increases.

\section{S8. Angular shift of the plasmon resonance upon $\mathrm{N}_{2} \mathrm{O}$ exposure}




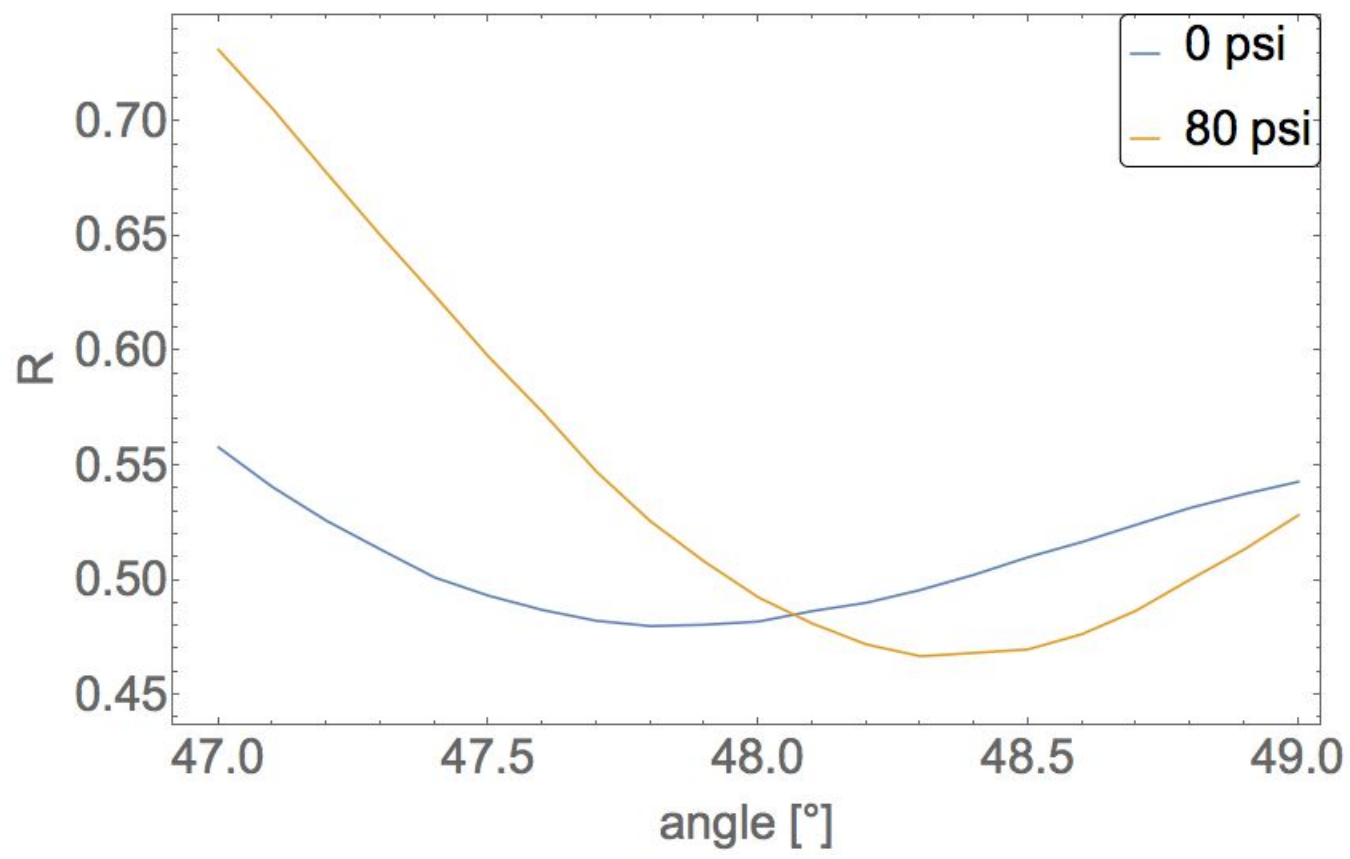

Figure S7. $\mathrm{N}_{2} \mathrm{O}$ pressure dependence of the angular shift of the maximum SPP absorption extracted at 2150 $\mathrm{cm}^{-1}$ for case $A$. The selected energy is off-resonance with the $\mathrm{N}_{2} \mathrm{O}$ absorber. These data correspond to vertical lines in the spectral maps presented throughout this manuscript. In this measurement mode, the CdO mid-IR plasmon exhibits the expected angular shift due to the change in refractive index in its environment. However, the angular shift does not convey any chemical (spectroscopic) information as to what caused the index change.

\section{S9. Pressure dependence of the modeled $\mathrm{N}_{2} \mathrm{O}$ dielectric function}
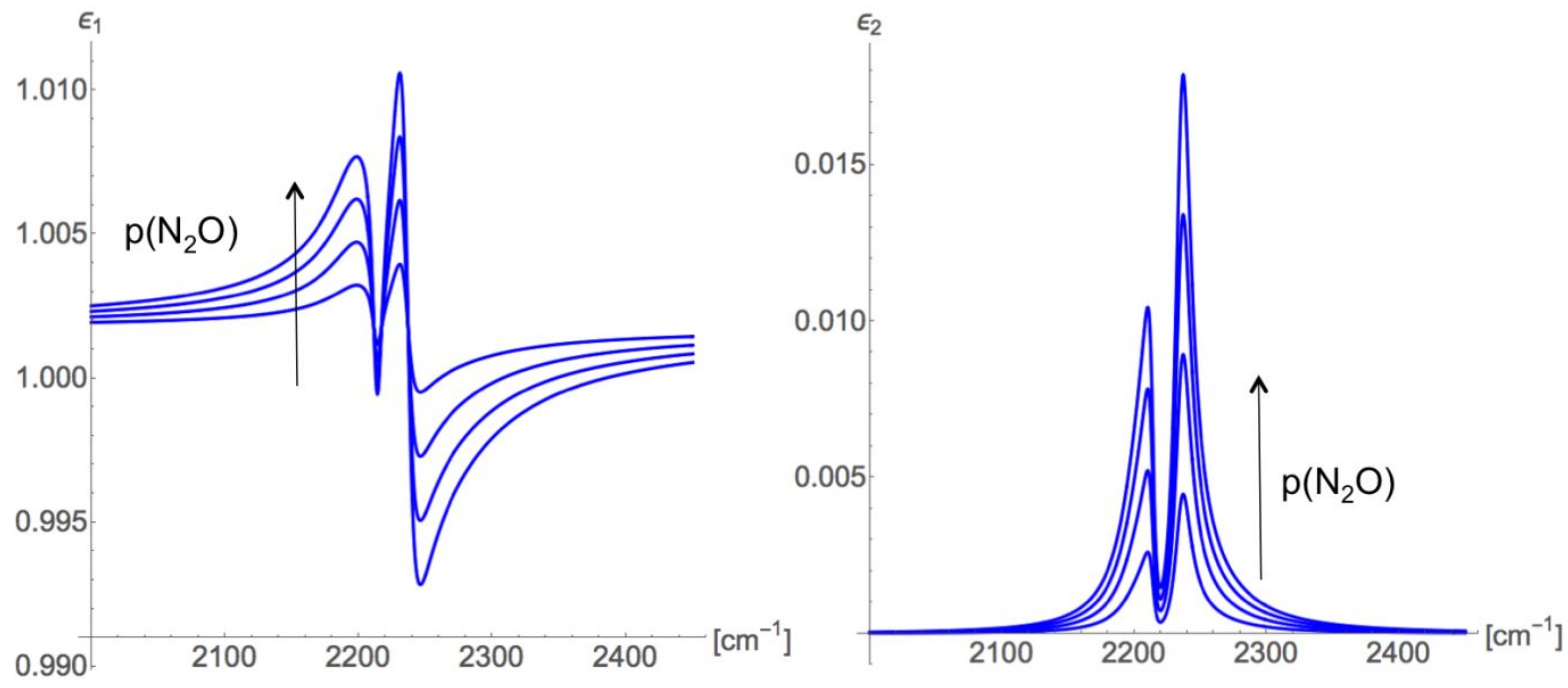

Figure S8. Pressure dependence of the dielectric function giving rise to $\mathrm{N}_{2} \mathrm{O}$ absorption. These dielectric functions were used for the substrate for all simulations with $\mathrm{N}_{2} \mathrm{O}$ present.

\section{References}


1. Stancik, A. L.; Brauns, E. B. A simple asymmetric lineshape for fitting infrared absorption spectra. Vibrational Spectroscopy 2008, 47 (1), 66-69.

2. $\quad$ Gordon, I. E.; Rothman, L. S.; Hill, C.; Kochanov, R. V.; Tan, Y.; Bernath, P. F.; Birk, M.; Boudon, V.; Campargue, A.; Chance, K. V.; Drouin, B. J.; Flaud, J. M.; Gamache, R. R.; Hodges, J. T.; Jacquemart, D.; Perevalov, V. I.; Perrin, A.; Shine, K. P.; Smith, M. A. H.; Tennyson, J.; Toon, G. C.; Tran, H.; Tyuterev, V. G.; Barbe, A.; Csaszar, A. G.; Devi, V. M.; Furtenbacher, T.; Harrison, J. J.; Hartmann, J. M.; Jolly, A.; Johnson, T. J.; Karman, T.; Kleiner, I.; Kyuberis, A. A.; Loos, J.; Lyulin, O. M.; Massie, S. T.; Mikhailenko, S. N.; Moazzen-Ahmadi, N.; Muller, H. S. P.; Naumenko, O. V.; Nikitin, A. V.; Polyansky, O. L.; Rey, M.; Rotger, M.; Sharpe, S. W.; Sung, K.; Starikova, E.; Tashkun, S. A.; Vander Auwera, J.; Wagner, G.; Wilzewski, J.; Wcislo, P.; Yu, S.; Zak, E. J. The HITRAN2016 molecular spectroscopic database. Journal of Quantitative Spectroscopy \& Radiative Transfer 2017, 203, 3-69. 